\title{
Prevalence of Zika virus neutralizing antibodies in healthy adults in Vietnam during and after the Zika virus epidemic season: a longitudinal population-based survey
}

Co Thach Nguyen ${ }^{1,2,3}$, Meng Ling Moi ${ }^{1,2,3^{*}}$, Thi Quynh Mai Le ${ }^{4}$, Thi Thu Thuy Nguyen ${ }^{4}$, Thi Bich Hau Vu ${ }^{4}$, Hai Tuan Nguyen ${ }^{4}$, Thi Thu Hang Pham ${ }^{4}$, Thi Hien Thu Le ${ }^{4}$, Le Manh Hung Nguyen ${ }^{5}$, Minh Huong Phu Ly ${ }^{1,2}$, Chris Fook Sheng $\mathrm{Ng}^{6}$, Taichiro Takemura ${ }^{7}$, Kouichi Morita ${ }^{1,2,3}$ and Futoshi Hasebe ${ }^{2,7}$

\begin{abstract}
Background: Between 2016 and 2019, 265 cases of Zika virus (ZIKV) infection were reported in Vietnam, predominantly in southern Vietnam. In 2016, a case of ZIKV-associated microcephaly was confirmed in the Central Highlands, and several members of the infant's family were confirmed to be infected with ZIKV. The study aims to determine the level of immunity to ZIKV in the general population of the ZIKV epidemic region.
\end{abstract}

Methods: A total of 879 serum samples were collected from 801 participants between January 2017 and July 2018, during and after the ZIKV epidemic in Vietnam. The samples were tested for anti-ZIKV immunoglobulin M (IgM) and immunoglobulin $\mathrm{G}(\mathrm{IgG})$, and anti-dengue virus (DENV) IgG antibodies using enzyme-linked immunosorbent assays (ELISA). Plaque-reduction neutralization test (PRNT) for ZIKV was performed on all samples, and for DENV on the samples that ZIKV neutralizing antibody positive.

Results: A total of 83 (10.3\%) participants had anti-ZIKV IgM. Of the 83, 6 were confirmed to be ZIKV antibodies positive using PRNT and anti-ZIKV IgG ELISA. Of the 718 participants who were anti-ZIKV IgM negative, a further 3 cases were confirmed as positive for antibodies against ZIKV. Of the 9 participants with ZIKV infection, 5 lived in the same village as the infant with ZIKV-associated microcephaly and the other 4 lived in 2 neighboring communes. Repeat samples were collected from the $83 \mathrm{ZIKV} \mathrm{IgM} \mathrm{positive} \mathrm{participants} 1.5$ years after the first collection. No new cases of ZIKV infection were detected. In addition, 2 of 3 participants with anti-ZIKV NS1 IgG demonstrated a 4- to 8 -fold increase in ZIKV neutralizing antibody titer.

(Continued on next page)

\footnotetext{
* Correspondence: sherry@nagasaki-u.ac.jp

${ }^{1}$ Graduate School of Biomedical Sciences, Nagasaki University, Nagasaki,

Japan

${ }^{2}$ Department of Virology, Institute of Tropical Medicine, Nagasaki University,

Sakamoto 1-12-4, Nagasaki 852-8523, Japan

Full list of author information is available at the end of the article
}

(c) The Author(s). 2020 Open Access This article is licensed under a Creative Commons Attribution 4.0 International License, which permits use, sharing, adaptation, distribution and reproduction in any medium or format, as long as you give appropriate credit to the original author(s) and the source, provide a link to the Creative Commons licence, and indicate if changes were made. The images or other third party material in this article are included in the article's Creative Commons licence, unless indicated otherwise in a credit line to the material. If material is not included in the article's Creative Commons licence and your intended use is not permitted by statutory regulation or exceeds the permitted use, you will need to obtain permission directly from the copyright holder. To view a copy of this licence, visit http://creativecommons.org/licenses/by/4.0/ The Creative Commons Public Domain Dedication waiver (http://creativecommons.org/publicdomain/zero/1.0/) applies to the data made available in this article, unless otherwise stated in a credit line to the data. 
(Continued from previous page)

Conclusions: ZIKV was present in the area around Krong Buk, with the rate of ZIKV-specific antibodies was $1.1 \%$ in the community since at least 2016. While the low levels of circulation together with low seroprevalence suggests a limited outbreak in the region, the results also reflect on low levels of protective immunity to Zika within the population. These results provide a better understanding of the current ZIKV epidemic status in the region and demonstrate a need for implementation of more effective ZIKV infection control measures.

Keywords: Zika virus, Zika virus infection, Seroprevalence, Seroepidemiologic studies, Neutralizing antibodies, Vietnam

\section{Background}

Zika virus (ZIKV) was first isolated from Rhesus monkeys in the Zika forest in Uganda in 1947 [1]. It is a mosquito-borne virus belonging to the genus Flavivirus and family Flaviviridae [2], which is spread from person to person mainly through the bite of infected Aedes aegepti and Aedes albopictus mosquitoes [3]. ZIKV can also be transmitted through sexual intercourse or body fluids [4]. Common symptoms are rash, fever, arthralgia, and conjunctivitis [5]. While ZIKV infection is sometimes associated with only mild symptoms, it can also lead to severe complications including Guillain-Barré syndrome [6] and microcephaly in infants [7].

The first reported ZIKV epidemic occurred in Yap Island, Federated States of Micronesia, in 2007, with an estimated 5000 of a total of 6800 residents infected [5]. The second reported epidemic occurred in French Polynesia in 2013 and 2014 , with an estimated 28,000 people infected, comprising approximately $11 \%$ of the population $[8,9]$. As many as 1.3 million people may have been infected in an epidemic in 14 states of Brazil in 2015 and 2016 [10]. During the epidemic period, there was an exponential increase in the number of cases of infants born with microcephaly suspected to be associated with ZIKV [7, 10]. According to a July 2019 WHO report there has been evidence of ZIKV transmission in 87 countries and territories in the Americas, Africa, Southeast Asia, and the Western Pacific region [11].

While Southeast Asia has been known as a ZIKV endemic region for more than 60 years, large ZIKV epidemics has only been reported recently [12]. Although the virus has been first isolated from mosquitoes in Malaysia in 1966, the first human cases were only reported in 1977 [13]. In 2016, a total of 455 cases were confirmed in Singapore [14] and, in Thailand, 386 cases were reported in 29 out of 76 provinces from 2015 to 2017 [15]. During this period, cases of ZIKV infection were also reported in other Southeast Asia nations including Malaysia [16] and Myanmar [17]. In 2016, 3 tourists were confirmed to have ZIKV infection after visiting Vietnam [18-20]. As of June 2019, a total of 265 cases has been reported in Vietnam, most of which occurred in Ho Chi Minh City [21-24]. Additionally, in 2016, a case of Zika-associated microcephaly was reported in the Central Highlands of Vietnam and 5 family members and 2 neighbors were confirmed positive for ZIKV infection [25]. Despite the endemicity for dengue and the high density of mosquito vectors, the numbers of cases of ZIKV infection in Vietnam remain substantially lower than the number of cases of dengue. Vietnam lies within the tropical zone where Aedes aegypti mosquitoes are endemic. While neighboring areas have reported ZIKV outbreaks in recent years, there are limited data available on the extent of ZIKV infection in local populations in Vietnam. Additionally, it has been hypothesized that dengue hyperendemicity may lead to crossreactive immunity toward ZIKV, thus limiting the size of ZIKV epidemics in Southeast Asia. However, there were limited seroprevalence data to support this hypothesis. Cross-reactivity between ZIKV and DENV antibodies has led to difficulties in the interpretation in some studies [26]. Annually, Vietnam reports approximately 100,000 dengue cases. The incidence of dengue has remained stable over the past few decades in Vietnam [27], and dengue seroprevalence remains high, with up to $64 \%$ of the adult population being seropositive [28]. Recent studies have suggested that while DENV is cross-reactive with ZIKV, the level of cross-neutralization and hence disease protection is limited [29-31]. In Vietnam, the number of ZIKV infections peaked at 219 in 2016, and has subsequently decreased, with only one reported case in 2019 [32]. The purpose of this study was to determine the seroprevalence of ZIKV antibodies among the population in Vietnam during and after the 2016 Zika epidemic using ZIKV and DENV neutralizing assays to elucidate the extent of the ZIKV epidemic in the local population.

\section{Methods}

\section{Ethics approval and consent to participate}

Ethics approval for this study was obtained from the ethics committee of the National Institute of Hygiene and Epidemiology, Ministry of Health, Vietnam (IRB-VN01057-45/ 2016) and the Ethics Committee of the Institute of Tropical Medicine, Nagasaki University, Japan (08061924-7).

\section{Sample collection}

The samples were collected from participants selected using simple random sampling by means of a lottery method in Krong Buk District, Dak Lak Province where a case of ZIKV-associated microcephaly had been 
reported [25]. The population density in this region is 181 people $/ \mathrm{km}^{2}$ with the estimated total population of 65,000 living in an area of $357.82 \mathrm{~km}^{2}$. In January 2017, 3 months after the case of microcephaly was reported, blood samples were collected from healthy adults in the community with places of residence distributed across all communes in Krong Buk District. None of the study participants were hospitalized for an acute illness during the study period. In this study, we estimated the proportion of persons with ZIKV infection in Krong Buk District.

The required sample size was calculated assuming a precision/absolute error (d) of $4 \%$ and a proportion with a 95\% level of confidence $\left(Z_{1-\alpha / 2}=1.96\right)$ [33]. Samples were processed within $24 \mathrm{~h}$ of collection and stored at $-80^{\circ} \mathrm{C}$ prior to testing.

\section{In-house Zika virus immunoglobulin M enzyme-linked immunosorbent assay}

The samples were screened for ZIKV IgM using an inhouse ZIKV IgM ELISA kit. This method was adapted from Dengue Virus IgM Capture DxSelect (Focus Diagnostics, Cypress, CA, USA) [34] by replacing DENV antigen with ZIKV antigen. The modified in-house ZIKV IgM ELISA has been utilized in other studies [25, 32, 35, 36]. Samples were first diluted 1:100 using sample diluent solution. The 96-well IgM Capture plate (Dengue Virus IgM Capture DxSelect, Focus Diagnostics) were soaked with 1X wash buffer solution for $5 \mathrm{~min}$, and wells were decanted. A total of $100 \mu \mathrm{L}$ of diluted serum sample was added and incubated at $37^{\circ} \mathrm{C}$ for $1 \mathrm{~h}$. The plates were then washed 3 times with $1 \mathrm{X}$ wash buffer solution. Next, $100 \mu \mathrm{L}$ of ZIKV antigen (MR-766, $10^{5} \mathrm{PFU} / \mathrm{mL}$ ) was added and incubated at room temperature (RT) for $1 \mathrm{~h}$. The wells were then washed with $1 \mathrm{X}$ wash buffer solution for a total of 3 times to remove excess antigen. Next, $100 \mu \mathrm{L}$ of affinity-purified and peroxidase-conjugated mouse anti-flavivirus antibodies was added to all wells and the plate was incubated at RT for $30 \mathrm{~min}$. The plates were washed 3 times to remove residual conjugate. Next, $100 \mu \mathrm{L}$ of tetramethylbenzidine (TMB) substrate solution and horseradish peroxide was added to each well and the plate was then incubated at RT for 10 mins in the dark. A total of $100 \mu \mathrm{L}$ of stop solution (1 M sulfuric acid) was added to each well to stop the reaction. Finally, the plates were read at $450 \mathrm{~nm}$ of the optical density (OD) using an ELISA plate reader (Multiscan ELISA reader, Thermolab System, Tokyo, Japan). OD values which were $\geq 2$ times that of the negative control $(\mathrm{N})$ is regarded as positive $(\mathrm{P})$.

\section{Detection of dengue virus immunoglobulins $M$ and $G$ (IgM and IgG), and Zika virus immunoglobulin-G by enzyme-linked immunosorbent assay (ELISA)}

In addition to the detection of anti-ZIKV IgM antibodies by ELISA, anti-dengue IgM antibodies (Vircell, Granada,
Spain) and anti-dengue IgG antibodies (Vircell) were determined according to manufacturer's instructions. Human Anti-Zika Virus IgG ELISA Kit (R\&D Systems, Inc. Minneapolis, MN, USA) was used to test samples for ZIKV-specific NS1-antigen-reactive IgG antibodies in the samples that exhibited anti-ZIKV IgM antibodies and neutralizing antibodies to ZIKV according to manufacturer's instructions. OD values which were $\geq 2$ times that of the negative control $(\mathrm{N})$ were regarded as positive $(\mathrm{P})$.

\section{Plaque-reduction neutralization test}

Serum samples were screened for the presence of neutralizing antibodies to ZIKV using a plaque-reduction neutralization test (PRNT). Serum samples were inactivated at $56^{\circ} \mathrm{C}$ for $30 \mathrm{~min}$ before testing. In the first PRNT screening for ZIKV, the serum was diluted 10 times in the EMEM (Nissui Pharmaceutical Co., Ltd., Tokyo, Japan) containing $2 \% \mathrm{FBS}$, then the serum samples were serially diluted 2-fold (1:10-1:10240). The PRNT was performed in replicates of 2 for ZIKV (MR766 laboratory strain) and all 4 DENV serotypes (DENV-1 01-44 strain; DENV-2 TLC-30 strain; DENV-3 CH53469 strain; DENV-4 SLMC318 strain). At each dilution, $50 \mu \mathrm{L}$ of serum sample was mixed with $50 \mu \mathrm{L}$ virus containing 100-200 plaque forming units (2000-4000 PFU/mL). The immune viruscomplex mixture was then incubated at $37^{\circ} \mathrm{C}$ for $1 \mathrm{~h}$. A total of $50 \mu \mathrm{L}$ of virus-immune complex mixture was then added onto BHK cell monolayers in 12 well plates (Corning Costar, Sigma-Aldrich, St. Louis, Missouri, USA) and incubated at $37^{\circ} \mathrm{C}$ in $5 \% \mathrm{CO}_{2}$ for $1 \mathrm{~h}$. After incubation, overlay medium ( $2 \mathrm{~mL}$ of EMEM/ $1 \%$ methylcellulose (Wako Pure Chemical Industries Ltd., Osaka, Japan), in 2\% FBS) was added into each well. The plates were incubated at $37^{\circ} \mathrm{C}$ in $5 \% \mathrm{CO}_{2}$ for $4-6$ days until visible plaque formation. Cells were then fixed using $4 \%$ paraformaldehyde phosphate buffer solution (Wako) for $1 \mathrm{~h}$ at RT and then stained with $1.25 \%$ crystal violet (Wako). The plaques were then counted by naked eye. The neutralization titers, $\mathrm{PRNT}_{50}$ and $\mathrm{PRNT}_{90}$, were defined as the highest serum dilution which reduced the number of plaques by 50 and $90 \%$ respectively. The presence of neutralizing antibodies was defined as ZIKV PRNT 50 titer $\geq 20$. The criteria to confirm the case exposed to ZIKV that has neutralizing antibodies to ZIKV with $\mathrm{PRNT}_{50}$ titer $\geq 20$, and 4 fold higher compare to DENV titers. The interpretation of probable ZIKV infection was according to WHO guidelines for laboratory testing for Zika virus infection in which samples that were positive for ZIKV IgM and negative for DENV IgM was interpreted as ZIKV probable case [37].

\section{Data analysis}

Descriptive analyses were performed, using frequencies and percentages for categorical variables; and means and standard deviations (SDs) for continuous variables. Odds 
ratios (ORs) and the 95\% confidence intervals (CIs) were used to estimate the relative likelihood of ZIKV infection in each group. Multiple logistic regression was used to assess the association of sex, ethnicity, and age group with ZIKV infection. The analyses were performed using Stata 14.1 (StataCorp LP, College Station, Texas, USA) with 5\% level of significance and two-tailed $p$ values were reported. The map was created using QGIS software 3.8.2.

\section{Results}

\section{Demographic characteristics of participants}

The serological surveillance study participants were randomly selected from the community of Krong Buk District. Serum samples were collected from 801 of 65,000 residents (1.2\%) in January 2017. The median age is 33 years, dominated by those in the age group between 16 and 60 years $(n=663$ or $82.7 \%)$. A total of 87 samples were collected from children under the age of 15 years $(10.9 \%)$. The proportion of females in the study was more than $73 \%$. In the analysis by ethnicity, 503 samples (62.8\%) were from the Kinh group, which is the major ethnic group in Vietnam, while a minority ethnic group Ede made up 290 samples (36.2\%), followed by 8 participants of other minor origin (1\%). The number of pregnant women who participated in this study was $66(8.24 \%)$.

\section{Zika virus and dengue seroprevalence}

Of the 801 samples tested, 83 (10.3\%) were positive for anti-ZIKV IgM antibodies (Table 1) with an average $\mathrm{P} / \mathrm{N}$ ratio of $3.30 \pm 1.48$. While the $\mathrm{P} / \mathrm{N}$ ratio is not a quantitative method, the ratio was used as a means to determine antibody levels of 83 samples, for the presence or absence of anti-ZIKV IgM antibodies. All participants who tested positive were asymptomatic at the time of sample collection. The prevalence of anti-ZIKV IgM antibodies varied moderately by age $(p=0.05)$ and was highest in the $46-$ 60 year age group (14.2\%) (Fig. 1(a)). The seroprevalence of ZIKV IgM did not differ significantly according to sex or ethnicity. Only one of the 66 pregnant women $(1.5 \%)$ was positive for anti-ZIKV IgM antibodies.

All 83 participants with ZIKV anti-IgM antibodies were negative for dengue IgM on ELISA; however, 49 (59.0\%) ZIKV IgM positive cases were detected as positive for Dengue IgG ELISA (Table 1). DENV IgM and DENV IgG antibodies was determined to examine possible cross-reactivity to ZIKV infection. The distribution of the ZIKV IgM antibodies positive cases, as well as DENV IgG antibodies positive cases, was observed in all age groups in this study (Fig. 1a). All testing results showed higher positive rates in the majority Kinh ethnic group than those in the minority Ede ethnic group (Fig. 1b).

\section{Neutralizing antibody levels to Zika virus and dengue virus serotypes 1-4}

All 801 samples were first screened to detect the presence of neutralizing antibodies to ZIKV. Of the 83 ZIKV IgM-positive samples, 8 demonstrated neutralizing antibodies to ZIKV $\left(\mathrm{PRNT}_{50}=1: 40\right.$ to $\left.1: 640\right)$. Of the 8 samples, 3 (Z141a, Z153a, Z735a) exhibited ZIKV antibody titers that were $>4$-fold higher than the antibody titers for all $4 \mathrm{DENV}$ serotypes $\left(\mathrm{PRNT}_{50} \mathrm{ZIKV}=1: 160\right.$ to 1: 640) (Table 2). The remaining 5 samples (Z120a,

Table 1 Demographic characteristics of the study participants $(N=801)$

\begin{tabular}{|c|c|c|c|c|c|c|c|c|}
\hline Variables & & $\mathrm{n}$ & $\begin{array}{l}\text { ZIKV IgM } \\
\text { positive (\%) }\end{array}$ & $\begin{array}{l}\text { Crude OR } \\
(95 \% \mathrm{Cl})\end{array}$ & $P$-value & $\begin{array}{l}\text { DENV IgG } \\
\text { positive (\%) } \\
(n=83)\end{array}$ & $\begin{array}{l}\text { Anti-ZIKV NS1 } \\
\text { IgG positive (\%) } \\
(n=88)\end{array}$ & $\begin{array}{l}\text { PRNT }_{50} \text { positive } \\
\text { to ZIKV (\%) }\end{array}$ \\
\hline \multirow[t]{6}{*}{ Age group (years) } & $\leq 15$ & 87 & $11(12.6)$ & 1.00 (Reference) & 0.05 & $4 / 11(36.3)$ & $3 / 11(27.3)$ & $2 / 87(2.3)$ \\
\hline & $16-30$ & 295 & $19(6.4)$ & $0.48(0.22-1.04)$ & & $9 / 19(47.4)$ & $3 / 21(14.3)$ & $3 / 295(1.0)$ \\
\hline & $31-45$ & 241 & $31(12.8)$ & $1.02(0.49-2.13)$ & & $18 / 31(58.1)$ & $7 / 32(21.9)$ & $3 / 241(1.2)$ \\
\hline & $46-60$ & 127 & $18(14.2)$ & $1.14(0.51-2.55)$ & & 14/18 (77.8) & 4/18 (22.2) & $1 / 127(0.8)$ \\
\hline & $\geq 60$ & 35 & $3(8.5)$ & $0.65(0.17-2.48)$ & & $3 / 3(100)$ & $1 / 5(20)$ & 3/35 (8.6) \\
\hline & $N A^{a}$ & 16 & $1(6.3)$ & & & $1 / 1(100)$ & $1 / 1(100)$ & $1 / 16(6.3)$ \\
\hline \multirow[t]{2}{*}{ Sex } & Female & 590 & $59(10)$ & 1.00 (Reference) & 0.57 & $32 / 59(54.2)$ & 10/64 (15.6) & $7 / 590(1.2)$ \\
\hline & Male & 211 & $24(11.4)$ & $1.15(0.7-1.91)$ & & 17/24 (70.8) & $9 / 24(37.5)$ & 6/211 (2.8) \\
\hline \multirow[t]{3}{*}{ Ethnicity } & Ede & 290 & $25(8.6)$ & 1.00 (Reference) & 0.16 & $15 / 25(60.0)$ & $5 / 26(19.2)$ & $3 / 290(1.0)$ \\
\hline & Kinh & 503 & $58(11.5)$ & $1.42(0.87-2.33)$ & & $34 / 58(58.6)$ & $14 / 62(22.6)$ & $10 / 503(2.0)$ \\
\hline & Other ${ }^{b}$ & 8 & 0 & & & & & 0/8 (0.0) \\
\hline Pregnancy & & 66 & $1(1.5)$ & & & $0 / 1(0.0)$ & $0 / 1(0.0)$ & $0 / 66(0.0)$ \\
\hline
\end{tabular}

Cl confidence interval, DENV dengue virus, IgG immunoglobulin G, IgM immunoglobulin M, NA not available, OR odds ratio, ZIKV Zika virus, ssIKV NS1 IgG positive, ZIKV antibodies were determined using anti-ZIKV NS1 IgG ELISA (P/N ratio $\geq 2$ ); PRNT $_{50}$ positive to ZIKV was defined as plaque-reduction neutralization test titers with $a \geq 50 \%$ reduction in ZIKV plaque-forming units at a serum dilution of $\geq 1: 20$

a Participants with missing information on age were excluded from the multivariate logistic regression analysis

b Participants whose ethnicity was categorized as "Other" were combined with the "Ede" ethnic group in the multivariate logistic regression analysis 

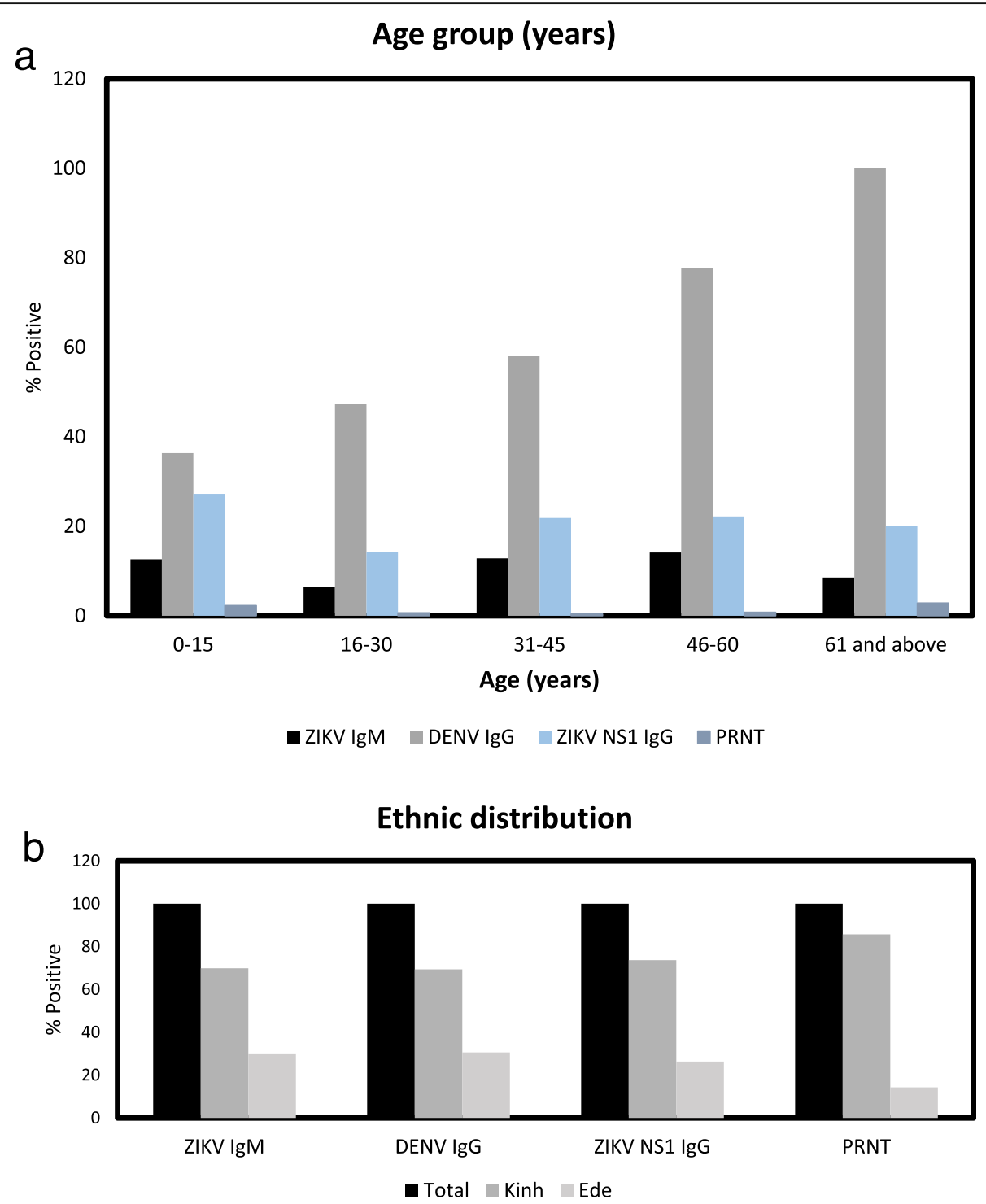

Fig. 1 Seroprevalence of Zika virus (ZIKV) antibodies in Central Vietnam, 2017-2018. The seroprevalence of Zika virus antibodies according to (a) age and (b) ethnicity. A total of 83 participants $(83 / 801,10.3 \%)$ demonstrated anti-ZIKV IgM antibodies as determined by using enzyme-linked immunosorbent assays. ZIKV neutralizing antibodies was determined by using plaque-reduction neutralization test (PRNT) and for confirming infection to ZIKV. Prevalence rates (anti-DENV IgG antibodies and anti-ZIKV NS1 IgG antibodies) was determined by using a total of 83 samples and 88 samples, respectively

Z138a, Z140a, Z420a, Z476a) were classified as probable ZIKV infections (PRNT ${ }_{50}$ ZIKV = 1: 40-1: 640).

Consecutive samples were collected from 78 of the 83 participants with anti-ZIKV IgM antibodies collected from the same participants 18 months after the first sample collection. Second consecutive samples were not collected from 5 participants who was not available at the time of second sample collection, including two (Z153 and Z420) of the 8 participants with ZIKV neutralizing antibodies in their initial sample. Two participants (Z120 and Z735) experienced a 2- to 4-fold decrease, and 2 participants (Z140 and Z476) experienced a 4- to 8-fold increase in their ZIKV neutralizing antibody titers (Table 2). Of the 83 participants with anti-ZIKV IgM antibodies, 5 (6.0\%; Z140, Z141, Z153, Z476 and Z735) had ZIKV neutralizing antibody titers that were at least 4-fold greater than their antibody titers against the 4 DENV serotypes tested.

Of the 718 samples that were negative for anti-ZIKV IgM antibodies, 5 (Z147a, Z472a, Z606a, Z587a and Z591a) demonstrated neutralizing antibodies to ZIKV (PRNT ${ }_{50}=$ 1:320-1:5120). Among the 5 samples, 2 samples (Z472a and Z606a) demonstrated a 4-fold or greater level of neutralizing antibodies to all 4 DENV serotypes (Table 2). In 


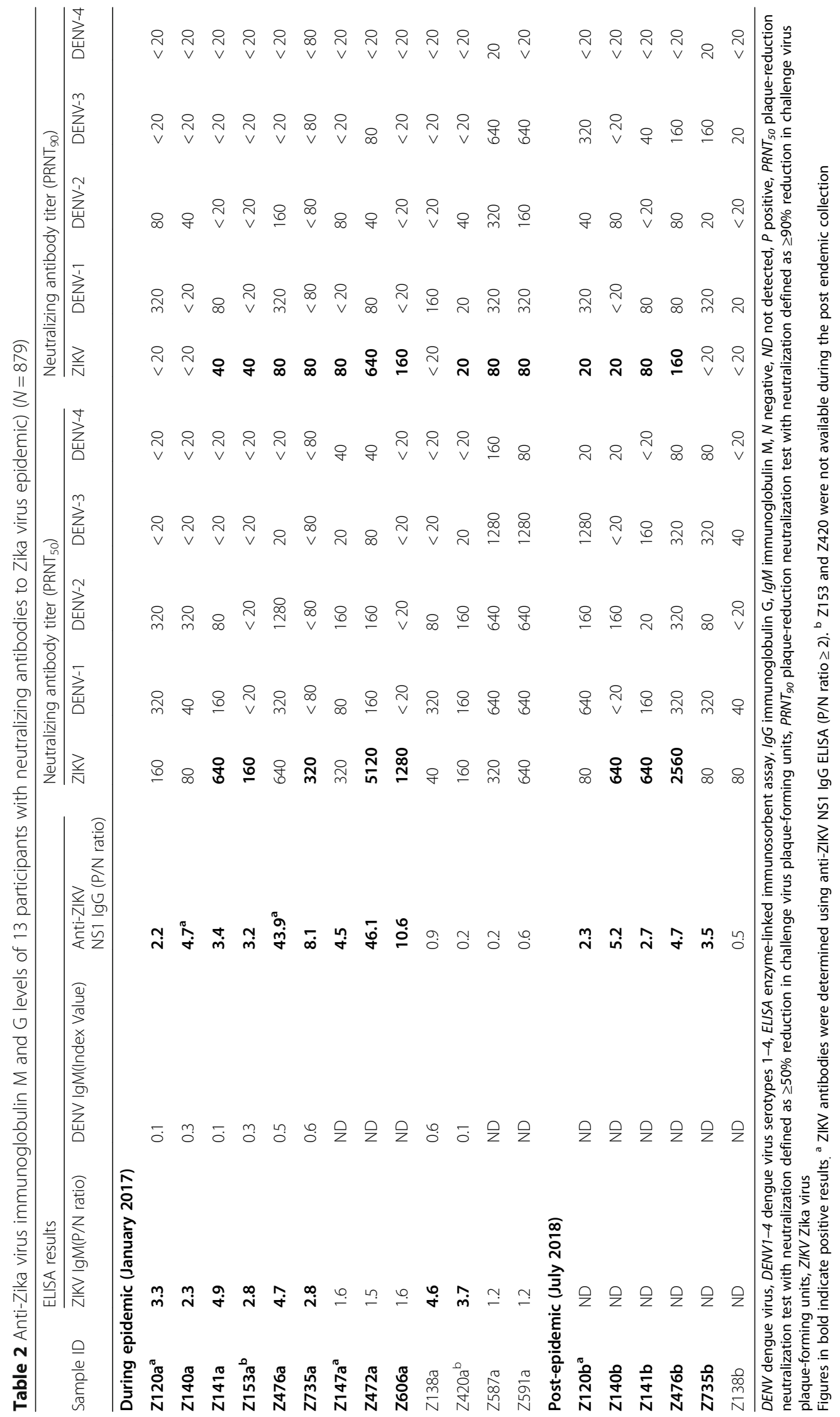


addition, by testing all 879 samples collected in both rounds using PRNT, 13 participants $(1.6 \%, N=801)$ had detectable ZIKV neutralizing antibodies. Overall, 7 participants $(0.9 \%$, Z140, Z141, Z153, Z476, Z735, Z472 and Z606) demonstrated ZIKV neutralizing antibody titers that were at least 4-fold higher than their antibody titers to all 4 DENV serotypes, whereas the other 6 participants $(0.8 \%)$ demonstrated comparable levels of ZIKV and DENV neutralizing antibodies. Thus, the results suggest that the 7 participants had been exposed to ZIKV during the 2016 ZIKV epidemic.

\section{Anti-Zika virus NS1 immunoglobulin G levels in cases of probable Zika virus infection}

Levels of anti-ZIKV NS1 IgG antibodies in the 83 samples that exhibited anti-ZIKV IgM antibodies and five samples that demonstrated ZIKV neutralizing antibodies but negative for anti-ZIKV IgM antibodies $(N=88)$ were determined using anti-Zika Virus NS1 IgG ELISA (R\&D Systems). Anti-ZIKV NS1 IgG assays are useful for confirming ZIKV infection because they are highly specific and possess minimal cross-reactivity to other flaviviruses [38]. Sixteen out of 83 ZIKV IgM positive samples (19.3\%) demonstrated ZIKV NS1 specific IgG antibodies, with a mean of $\mathrm{P} / \mathrm{N}$ ratio of $5.7 \pm 10.3$ (data not shown). Among 8 of 83 ZIKV IgM positive samples that demonstrated neutralizing antibodies to ZIKV, 6 samples (Z120a, Z140a, Z141a, Z153a, Z476a and Z735a) were also positive for anti-ZIKV NS1 IgG by using ELISA. All of 3 samples (Z141a, Z153a and Z735a) that demonstrated a 4-fold or greater ratio of ZIKV neutralizing antibody titers to DENV antibody titers also demonstrated ZIKV NS1 IgG antibodies $(\mathrm{P} / \mathrm{N}$ ratio $=3.1-8.1$ ). These results confirm that these 3 participants were exposed to ZIKV infection. Three of the 5 samples (Z120a, Z138a, Z140a, Z420a, Z476a) with ZIKV neutralizing antibodies were also confirmed positive for anti-ZIKV NS1 antibodies. The Anti-ZIKV NS1 IgG levels in the second samples collected in July 2018 were comparable to the levels in the first samples.

Three of 5 samples (Z147, Z472, Z587, Z591 and Z606) that demonstrated ZIKV neutralizing antibodies $(N=718$, ZIKV IgM negative samples) were also positive for antiZIKV NS1 IgG antibodies. Two samples (Z472 and Z606) were positive for both ZIKV neutralizing antibodies and ZIKV NS1 IgG $(\mathrm{P} / \mathrm{N}$ ratio $=10.6$ and 46.1 respectively). In addition, one sample (Z147) was also positive for anti-NS1 antibodies with a $\mathrm{P} / \mathrm{N}$ ratio of 4.5 . Finally, a total of 19 samples demonstrated anti-ZIKV NS1 IgG antibodies, including 16 of 83 samples that exhibited anti-ZIKV IgM antibodies positive and three samples that showed ZIKV neutralizing antibodies but negative for anti-ZIKV IgM antibodies (Table 1). However, out of the 19 samples that were positive for anti-ZIKV NS1 IgG antibodies, only 9 (Z120, Z140, Z141, Z153, Z476, Z735, Z147, Z472 and
Z606) exhibited neutralizing antibodies against ZIKV with $\mathrm{PRNT}_{50}$ titers $\geq 1: 20$.

In summary, out of 801 participants tested in this study, by using two methods: the anti-NS1 IgG ELISA and PRNT, we determined that 9 participants $(1.12 \%$, Z120, Z140, Z141, Z147, Z153, Z735, Z476, Z472 and Z606) had ZIKV infection. Of these 9 participants, 5 (Z120, Z140, Z141, Z147 and Z153) lived in Cu Pong commune. An infant with microencephaly and her immediate family members has been confirmed confirmed positive for ZIKV infection in the Cu Pong commune in 2016 [25]. This study however excludes samples from our previous study [25]. The 4 remaining cases ( $Z 472$, Z476, Z606 and Z735) were found in 2 neighboring communes, two cases in Chu Kbo (1.7\%, Z472 and Z476) and two other cases in Pong Drang village (0.99\%) (Fig. 2).

\section{Discussion}

In this study, we determined the anti-ZIKV IgM and IgG antibodies among 801 participants who were recruited during ZIKV epidemic in Vietnam. Of the 83 first samples that demonstrated ZIKV IgM antibodies, only $8(1.0 \%)$ had ZIKV neutralizing antibodies (PRNT = 1:40-1:640) with 3 cases exposed to ZIKV infection confirmed using $\mathrm{PRNT}_{50}$ with ZIKV neutralizing titer $\geq 4$ fold of DENVs neutralizing titer. While anti-IgM ZIKV antibodies may have lower specificity than other laboratory assays, the test is useful for identifying probable ZIKV cases during an outbreak [32, 39]. Due to relatively short detection window for ZIKV IgM antibodies, a combination of tests that determine ZIKV neutralizing antibodies and ZIKV-specific IgG antibodies would be useful for long-term seroprevelance studies. As such, ZIKV IgM test was used to determine ZIKV IgM antibodies during the ZIKV outbreak in Vietnam in 2016, and ZIKV-specific IgG ELISA and PRNT was included in this study. In further tests by using the anti-ZIKV NS1 IgG test, 3 of the 5 probable cases were confirmed positive for anti-ZIKV NS1 IgG antibodies. By using both PRNT (ZIKV, DENV1-4) and anti-ZIKV NS1 IgG assays in all 83 participants that demonstrated crossreactive ZIKV IgM antibodies, only $6(7.2 \%)$ samples fulfilled the criteria of ZIKV PRNT ratio $>4$ and presence of ZIKV-specific NS1 cross-reactive antibodies, confirming that these 6 individuals had recently become infected with ZIKV. Using samples from the same participants, the levels of antibodies were determined 18 months after the first sample collection (post-ZIKV epidemic). Among these 6 confirmed cases, 3 samples that were only ZIKV NS1 IgG antibodies positive during the first collection also demonstrated high ZIKV neutralization titers 18 months later. None of the ZIKV seropositive participants 


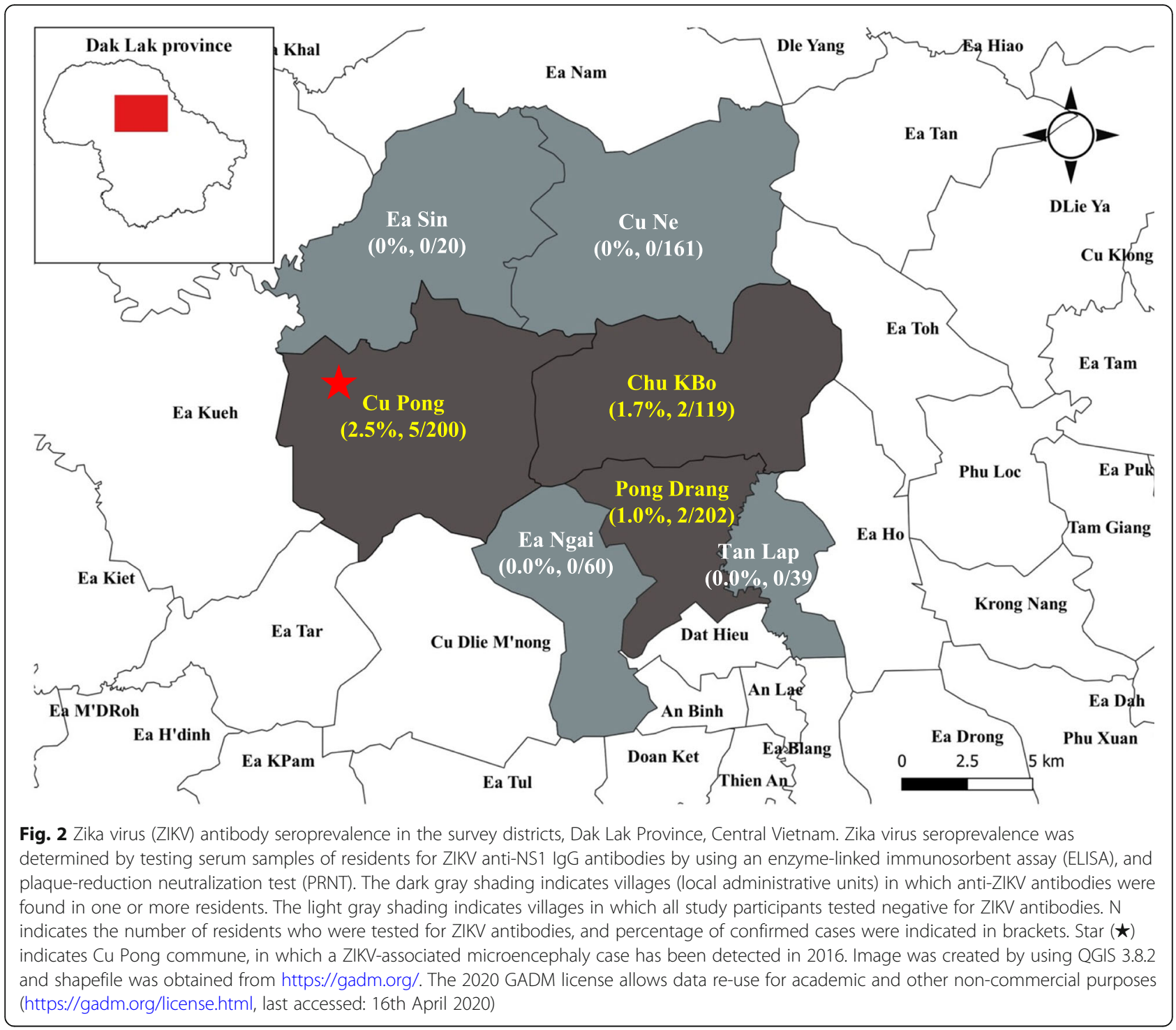

had an international travel history, suggesting that local transmission in the area.

While the prevalence of anti-ZIKV IgM antibodies was highest in the 46-60 year age group, there were no significant discrepancy in anti-ZIKV IgM seropositivity rates across age groups, indicating that the risk of ZIKV infection is homologous across different age groups. In contrast, a high proportion of participants demonstrated DENV IgG antibodies, with seropositivity increasing with age $[37,40,41]$. These results are consistent with those of other studies, indicating association with longer exposure due to persistent DENV endemicity. While there were high levels of DENV seropositivity (49/83; 59.0\%), indicating DENV exposure in the community, the overall seropositive rates for ZIKV remains low $(9 / 801 ; 1.1 \%)$. While low ZIKV seroprevalence, a proxy of protection, indicates vulnerability of the population of the region to the ZIKV epidemic, the results also suggest that DENV may offer limited cross-protection against ZIKV.

Among the participants, there was no significant difference in ZIKV IgM seroprevalence according to sex. The higher percentage of female participants in this study is due to socioeconomic factors in this region, as adult males have a higher tendency to travel for employment opportunities in urban areas. While statistically insignificant, the proportion of ZIKV IgM seropositivity in the Kinh ethnic group was higher (11.5\%) than that of the minority Ede ethnic group (8.6\%). This tendency may be due to the Kinh ethic group possessing higher proficiency in the national language, and are thus, more likely to travel and had higher mobility as compared to other minority ethnic groups. This may reflect as increased risk of 
infectious disease exposure due to ethnic and socioeconomical associated activity and behaviors. However, in the context of local socioeconomic development, rural poverty is still predominant, particularly in terms of access to medical care. These socioeconomic factors may further drive inter-city migrant workers to travel between larger cities and rural areas, in addition to low seroprevalence rate in the region, these factors may in turn lead to further Zika epidemic expansion during outbreaks.

Of the 718 samples that were negative for ZIKV IgM antibodies, 5 samples demonstrated high levels of ZIKV neutralizing antibodies with titers from 1: 320-1: 5120 . Additionally, 3 samples were also positive for anti-ZIKV NS1 IgG, 2 of these 3 samples were confirmed by PRNTs with neutralizing antibody titers of 1:1280-1: 5120. These results indicate local ZIKV transmission within the healthy community in the Central Highlands of Vietnam. Overall, 9/801 (1.1\%) of the ZIKV confirmed case as positive for ZIKV had 5/200 (2.5\%) cases collected in $\mathrm{Cu}$ Pong village, the same commune with the case of microcephaly cases that we reported in the previous study. In the vicinity at Chu Kbo and Pong Drang villages, only $2 / 119(1.68 \%)$ and $2 / 202(0.99 \%)$ positive cases were identified, respectively. This result indicates that the circulation of ZIKV in Central Vietnam is limited in the period of 2 years since the confirmation of nationwide ZIKV outbreak in Vietnam. In comparison with previous reports in Indochina, the ZIKV seroprevalence found in this study is at comparable levels with those of Cambodia [42] and Laos [43]. The low ZIKV seroprevalence, a proxy of protection against the disease in the community, however indicates that the community is at risk of subsequent ZIKV epidemic.

\section{Conclusion}

This study confirms ZIKV infection in the Central Highlands of Vietnam and suggests that ZIKV has been present in the province since at least 2016. The prevalence of ZIKV-specific antibodies was $1.1 \%$ at the start of the study period, suggesting a limited outbreak within the area. Economic factors including migrant workers may play a role in introducing emerging pathogens such as ZIKV to rural areas. While DENV seroprevalence remains high in the region, the overall low ZIKV seroprevalence indicate limited Zika disease protection in the population. Further studies of seroprevalence in the general population and continuous surveillance are needed to better understand the extent of the outbreak in the general population and to define the potential risk of ZIKV transmission in the region.

\section{Abbreviations}

DENV: Dengue virus; ELISA: Enzyme-linked immunosorbent assay; IgG: Immunoglobulin G; IgM: Immunoglobulin M; PRNT: Plaque-reduction neutralization test; ZIKV: Zika virus

\section{Acknowledgments}

We would like to thank Drs. Tho Duoc Pham, Ngoc Thanh Pham, and Van Tuan Le of the Tay Nguyen Institute of Hygiene and Epidemiology, Buon Ma Thuot, Dak Lak, Vietnam; and Dr. Thuan Tran of Preventive Medicine Center, Krong Buk, Dak Lak, Vietnam, for helping with sample collection. We would also like to thank to Mr. Ngoc Linh Nguyen and Mr. Do Quyen Pham of the National Institute of Hygiene and Epidemiology, Hanoi, Vietnam for providing technical assistance and support; and members of the Department of Virology, Institute of Tropical Medicine (NEKKEN), Nagasaki University, Nagasaki, Japan for providing technical advice and support. The first author (CTN) is grateful for the academic support from the Program for Nurturing Global Leaders in Tropical and Emerging Communicable Diseases, Graduate School of Biomedical Sciences, Nagasaki University.

\section{Authors' contributions}

CTN carried out the ELISA, PRNTs, performed data analyses, and drafted the manuscript. MLM carried out part of the ELISA and PRNT tests, performed data analyses, contributed reagents and materials, conceived the study, participated in study design and drafted the manuscript. FH, and KM contributed reagents and materials, conceived the study and, participated in study design and coordination. TQML, TTTN contributed reagents, study design and coordination, and sample collection. TBHV, TTHP, THTL, TT contributed to screening samples by ELISA and molecular diagnosis. LMHN performed calculations in the sample size and sample collection. MHPL, CNFS, and HTN contributed to statistical analysis. All authors have read and approved the final manuscript.

\section{Funding}

This research was supported by Japan Agency for Medical Research and Development (AMED) under Grant Number JP19fm0108001(Japan Initiative for Global Research Network on Infectious Diseases (J-GRID), AMED JP20wm0125006; and AMED Research on Emerging and Re-emerging Infectious Diseases (19fk0108109h0001, 20fk0108109h0001, 20fk0108123h1101) and Nagasaki University. The funders had no role in study design, data collection and analysis, decision to publish, or preparation of the manuscript. CTN was a recipient of the Japanese government (Monbukagakusho) Scholarship.

\section{Availability of data and materials}

The datasets used and/or analyzed during the current study are available from the corresponding author on reasonable request.

\section{Ethics approval and consent to participate}

Ethics approval for this study was obtained from the ethics committee of the National Institute of Hygiene and Epidemiology, Ministry of Health, Vietnam (IRB-VN01057-45/2016) and the Ethics Committee of the Institute of Tropical Medicine, Nagasaki University, Japan (08061924-7). The study obtained written consent from the study participants and the parents of participants under 16 years of age after the objectives and the procedures was explained accordingly.

\section{Consent for publication}

Not applicable.

\section{Competing interests}

The authors declare that they have no competing interests.

\footnotetext{
Author details

${ }^{1}$ Graduate School of Biomedical Sciences, Nagasaki University, Nagasaki, Japan. ${ }^{2}$ Department of Virology, Institute of Tropical Medicine, Nagasaki University, Sakamoto 1-12-4, Nagasaki 852-8523, Japan. ${ }^{3}$ Program for Nurturing Global Leaders in Tropical and Emerging Communicable Diseases, Nagasaki University, Nagasaki, Japan. ${ }^{4}$ National Institute of Hygiene and Epidemiology, Hanoi, Vietnam. ${ }^{5}$ Tay Nguyen Institute of Hygiene and Epidemiology, Tay Nguyen, Dak Lak, Vietnam. ${ }^{6}$ School of Tropical Medicine and Global Health, Nagasaki University, Nagasaki, Japan. 'Vietnam Research Station, Center for Infectious Disease Research in Asia and Africa, Institute of Tropical Medicine, Nagasaki University, Nagasaki, Japan.
} 
Received: 5 February 2020 Accepted: 16 April 2020

Published online: 11 May 2020

\section{References}

1. Dick GW, Kitchen S, Haddow A. Zika virus (I). Isolations and serological specificity. Trans R Soc Trop Med Hyg. 1952;46:509-20. https://doi.org/10. 1016/0035-9203(52)90042-4.

2. Kuno G, Chang G-JJ, Tsuchiya KR, Karabatsos N, Cropp CB. Phylogeny of the Genus Flavivirus. 1998. https://www.ncbi.nlm.nih.gov/pmc/articles/PMC1 09351/pdf/jv000073.pdf. Accessed 26 Dec 2018.

3. Boyer S, Calvez E, Chouin-Carneiro T, Diallo D, Failloux A-B. An overview of mosquito vectors of Zika virus. Microbes Infect. 2018;20:646-60. https://doi. org/10.1016/J.MICINF.2018.01.006.

4. Zamani M, Zamani V. Sexual transmission of Zika virus: an assessment of the evidence. Iran J Public Health. 2017;46:1305-6 http://www.ncbi.nlm.nih.gov/ pubmed/29026802.

5. Duffy MR, Chen T-H, Hancock WT, Powers AM, Kool JL, Lanciotti RS, et al. Zika virus outbreak on Yap Island, Federated States of Micronesia. N Engl J Med. 2009;360:2536-43. https://doi.org/10.1056/NEJMoa0805715.

6. Kandel N, Lamichhane J, Tangermann RH, Rodier GR. Detecting GuillainBarré syndrome caused by Zika virus using systems developed for polio surveillance. Bull World Health Organ. 2016;94:705-8. https://doi.org/10. 2471/BLT.16.171504.

7. Teixeira MG, da CN CM, de Oliveira WK, Nunes ML, Rodrigues LC. The Epidemic of Zika Virus-Related Microcephaly in Brazil: Detection, Control, Etiology, and Future Scenarios. Am J Public Health. 2016;106:601-5. https:// doi.org/10.2105/AJPH.2016.303113.

8. Besnard M, Lastère S, Teissier A, Cao-Lormeau V, Musso D. Evidence of perinatal transmission of Zika virus, French Polynesia, December 2013 and February 2014. Eurosurveillance. 2014;19:20751. https://doi.org/10.2807/ 1560-7917.ES2014.19.13.20751.

9. Musso D, Nilles EJ, Cao-Lormeau V-M. Rapid spread of emerging Zika virus in the Pacific area. Clin Microbiol Infect. 2014;20:0595-6. https://doi.org/10. 1111/1469-0691.12707

10. Petersen LR, Jamieson DJ, Powers AM, Honein MA. Zika Virus. N Engl J Med. 2016:374:1552-63. https://doi.org/10.1056/NEJMra1602113.

11. WHO |Zika epidemiology update. WHO. 2019. https:/www.who.int/ emergencies/diseases/zika/epidemiology-update/en/. Accessed 14 Oct 2019

12. Wiwanitkit $V$. The current status of Zika virus in Southeast Asia. Epidemiol Health. 2016;38:e2016026. https://doi.org/10.4178/epih.e2016026.

13. Olson JG, Ksiazek TG, Suhandiman T. Zika virus, a cause of fever in Central Java, Indonesia. Trans R Soc Trop Med Hyg. 1981;75:389-93. https://doi.org/ 10.1016/0035-9203(81)90100-0.

14. Ho ZJM, Hapuarachchi HC, Barkham T, Chow A, Ng LC, Lee JMV, et al. Outbreak of Zika virus infection in Singapore: an epidemiological, entomological, virological, and clinical analysis. Lancet Infect Dis. 2017;17: 813-21. https://doi.org/10.1016/S1473-3099(17)30249-9.

15. Ruchusatsawat K, Wongjaroen P, Posanacharoen A, Rodriguez-Barraquer I, Sangkitporn S, Cummings DAT, et al. Long-term circulation of Zika virus in Thailand: an observational study. Lancet Infect Dis. 2019;19:439-46. https:// doi.org/10.1016/S1473-3099(18)30718-7.

16. Woon YL, Lim MF, Tg Abd Rashid TR, Thayan R, Chidambaram SK, Syed Abdul Rahim SS, et al. Zika virus infection in Malaysia: an epidemiological, clinical and virological analysis. BMC Infect Dis. 2019;19:152. https://doi.org/ 10.1186/s12879-019-3786-9.

17. Myat Ngwe Tun M, Kyaw Kyaw A, Wut Hmone S, Inoue S, Buerano CC, Min Soe A, et al. Detection of Zika Virus Infection in Myanmar. Am J Trop Med Hyg. 2018;98:SS. https://doi.org/10.4269/ajtmh.17-0708.

18. Meltzer E, Lustig Y, Leshem E, Levy R, Gottesman G, Weissmann R, et al. Zika virus disease in traveler returning from Vietnam to Israel. Emerg Infect Dis. 2016;22:1521-2. https://doi.org/10.3201/eid2208.160480.

19. Katanami Y, Kutsuna S, Taniguchi S, Tajima S, Takaya S, Yamamoto K, et al. Detection of Zika virus in a traveller from Vietnam to Japan. J Travel Med. 2017;24. https://doi.org/10.1093/jtm/tax031.

20. Lim SK, Lim JK, Yoon IK. An Update on Zika Virus in Asia. Infect Chemother. 2017:49:91-100. https://doi.org/10.3947/ic.2017.49.2.91.

21. Hashimoto T, Kutsuna S, Tajima S, Nakayama E, Maeki T, Taniguchi S, et al. Importation of Zika virus from Vietnam to Japan, November 2016. Emerg Infect Dis. 2017;23:1223. https://doi.org/10.3201/EID2307. 170519.
22. Lan PT, Quang LC, Huong VTQ, Thuong NV, Hung PC, Huong TTLN, et al. Fetal Zika virus infection in Vietnam. PLoS Curr. 2017;9. https://doi.org/10. 1371/currents.outbreaks.1c8f631e0ef8cd7777d639eba48647fa.

23. Quyen NTH, Kien DTH, Rabaa M, Tuan NM, Vi TT, Van Tan L, et al. Chikungunya and Zika virus cases detected against a backdrop of endemic dengue transmission in Vietnam. Am J Trop Med Hyg. 2017;97:146-50. https://doi.org/10.4269/ajtmh.16-0979.

24. Bui TC, Huynh XV, Nguyen H, Nguyen KTT, Nguyen CD, Pham ND. Zika virus outbreaks and treatment in pregnant women in Ho Chi Minh City, Vietnam. Int J Gynecol Obstet. 2018;141:390-1.

25. Moi ML, Nguyen TT, Nguyen CT, Vu TBH, Tun MMN, Pham TD, et al. Zika virus infection and microcephaly in Vietnam. Lancet Infect Dis. 2017;17:8056. https://doi.org/10.1016/S1473-3099(17)30412-7.

26. Hayes EB. Zika virus outside Africa. Emerg Infect Dis. 2009;15:1347-50. https://doi.org/10.3201/eid1509.090442.

27. Hung TM, Clapham HE, Bettis AA, Cuong HQ, Thwaites GE, Wills BA, et al. The estimates of the health and economic burden of dengue in Vietnam. Trends Parasitol. 2018;34:904-18. https://doi.org/10.1016/J.PT.2018.07.007.

28. Thai KTD, Binh TQ, Giao PT, Phuong HL, Hung LQ, Van Nam N, et al. Seroprevalence of dengue antibodies, annual incidence and risk factors among children in southern Vietnam. Trop Med Int Heal. 2005;10:379-86. https://doi.org/10.1111/j.1365-3156.2005.01388.x

29. Musso D, Gubler DJ. Zika Virus. Clin Microbiol Rev. 2016;29:487-524. https:// doi.org/10.1128/CMR.00072-15.

30. Oliveira RA, de Oliveira-Filho EF, Fernandes Al, Brito CA, Marques ET, Tenório MC, et al. Previous dengue or Zika virus exposure can drive to infection enhancement or neutralisation of other flaviviruses. Mem Inst Oswaldo Cruz. 2019;114:1-7.

31. Heinz FX, Stiasny K. The antigenic structure of Zika virus and its relation to other Flaviviruses: implications for infection and Immunoprophylaxis. Microbiol Mol Biol Rev. 2017;81. https://doi.org/ 10.1128/MMBR.00055-16

32. Wijesooriya SL, Nguyen CT, Nguyen TT, Vu TBH, Taichiro T, Morita K, et al, Long-term surveillance needed to detect Zika virus outbreaks in endemic regions. Lancet Infect Dis. 2020;20:168-9. https://doi.org/10.1016/S14733099(19)30677-2.

33. Pourhoseingholi MA, Vahedi M, Rahimzadeh M. Sample size calculation in medical studies. Gastroenterol Hepatol from bed to bench. 2013;6:14-7 http://www.ncbi.nlm.nih.gov/pubmed/24834239. Accessed 22 Oct 2019.

34. Focus Diagnostics. Dengue Virus IgM Capture DxSelect TM (OUS). http:// www.focusdx.com/pdfs/pi/OUS/EL1500M-OUS.pdf. Accessed 28 Dec 2018.

35. Kutsuna S. Zika virus infection: clinical overview with a summary of Japanese cases. Clin Exp Neuroimmunol. 2017;8:192-8. https://doi.org/10. $1111 /$ cen3.12408.

36. Martin DA, Muth DA, Brown T, Johnson AJ, Karabatsos N, Roehrig JT. Standardization of immunoglobulin M capture enzyme-linked immunosorbent assays for routine diagnosis of arboviral infections. J Clin Microbiol. 2000;38:1823-6 http://www.ncbi.n/m.nih.gov/pubmed/10790107. Accessed 12 Mar 2020

37. Pham HV, Doan HT, Phan TT, Tran Minh NN. Ecological factors associated with dengue fever in a central highlands province. Vietnam BMC Infect Dis. 2011;11:172. https://doi.org/10.1186/1471-2334-11-172.

38. R\&D Systems. Human Anti-Zika Virus lgG ELISA Kit ZIKO0. https://www.rndsystems. com/products/human-anti-zika-virus-igg-elisa-kit_zik00. Accessed 3 Feb 2020.

39. WHO. Laboratory testing for Zika virus infection. World Heal Organ. 2016;1. https://doi.org/10.1038/sj.mp.4001048.

40. Van Le T, Thi Tuyet Van N, Hoang Quan N, Tho DP. Phylogeny of dengue virus type 2 isolated in the central highlands. Vietnam Rev Biol Trop. 2017;65:819-26.

41. Van Tuan L, Van NTT, Thi P, Nga T, Duong L, Quan M, et al. Seasonal distribution of dengue fever in the central highlands region, Vietnam (20102015). Am J Epidemiol Infect Dis. 2017;5:8-13.

42. Duong V, Ong S, Leang R, Huy R, Ly S, Mounier U, et al. Low circulation of Zika virus, Cambodia, 2007-2016. Emerg Infect Dis. 2017;23:296-9. https:// doi.org/10.3201/eid2302.161432..

43. Pastorino B, Sengvilaipaseuth $\mathrm{O}$, Chanthongthip A, Vongsouvath $M$, Souksakhone C, Mayxay M, et al. Low Zika virus Seroprevalence in Vientiane, Laos, 2003-2015. Am J Trop Med Hyg. 2019;100:639-42. https://doi.org/10. 4269/ajtmh.18-0439.

\section{Publisher's Note}

Springer Nature remains neutral with regard to jurisdictional claims in published maps and institutional affiliations. 